\title{
The effects of checkerboard pattern check size on the VECP
}

\author{
JOHN B. SIEGFRIED \\ Pennsylvania College of Optometry, Philadelphia, Pennsylvania 19141
}

\begin{abstract}
Studies have shown that there is an optimal size of element in a checkerboard pattern, used to stimulate the visual system, for eliciting the visual evoked cortical potential (VECP). This size of element is between 8 and $25 \mathrm{~min}$ of visual angle. Since most studies have not reported fixation or location of fixation on the pattern, this study was performed in which fixation was specified at a location which ensures a constant illumination of the fovea regardless of check size. The resulting function, VECP amplitude vs. check size, resembles previously obtained functions, indicating either that previous subjects were fixating or that fixation is not important for these results.
\end{abstract}

Recent studies of the visual evoked cortical potential (VECP) have begun moving away from the use of large, homogeneous fields of light used to illuminate a substantial portion of man's heterogeneous retina. Instead, increasing emphasis is being placed on focal stimulation, and on patterning of this focal stimulation. Most of the work on simple black and white checkerboard-patterned stimuli has shown that such structured stimuli, when flashed repetitively on the retina, produce averaged VECP of considerably greater amplitude than those evoked by nonpatterned stimulus input. One focus of interest has been the dependence of the amplitude of the VECP upon the size of the "check" in the checkerboard pattern used to evoke the potential. It has been found that the function relating amplitude of VECP to check size is not monotonic, but, rather, exhibits a maximum in the region of from 8 to $25 \mathrm{~min}$ of visual angle (the angle subtended on the retina by the side of one check in the pattern).

In the most comprehensive sets of experiments on the effect of various parameters of the checkerboard pattern on the VECP (Rietveld, Tordoir, Hagenouw, Lubbers, \& Spoor, 1967; Spehlmann, 1965), as well as in some experiments by others, no mention is made of whether the observer was required to fixate upon an added fixation target or upon a specific feature of the checkerboard pattern itself; and if the observer was indeed required to fixate, some other reports fail to mention where that point of fixation was located with respect to certain details of the pattern (Ciganek, 1971; Harter, 1971; Harter \& White, 1968, 1970). The exact location of the observer's point of fixation with respect to the checkerboard pattern could be of crucial importance in some experimental situations. In their publications relating to the effect of check size on the VECP, most investigators are quick to point out that since all of their patterns have equal light and dark areas, the total light flux produced by the stimulus is therefore independent of check size (field size remaining constant). I have no argument with this fact. But consider the consequences of various locations of fixation for the amount of light flux reaching the fovea, which is the major input for the VECP. In the case of a large-check pattern, in which one or a few checks might subtend a visual angle of greater than $1 \mathrm{deg}$, the fovea would be receiving vastly different amounts of illumination, depending on whether the observer was fixating the center of a light check or the center of a dark check, even though the total amount of flux entering the eye is the same in either case.

It seemed to me, therefore, to be worth repeating the experiment on the effect of check size upon the amplitude of the VECP with a mode of fixation which assured a more nearly constant illumination of the fovea across all patterns used, and to obtain the function in sufficient detail so that it could be compared with the highly detailed functions of Rietveld et al. (1967). In the data to be reported here, fixation was upon the point formed by the intersection of four checks (two light and two dark). In this manner, light checks always illuminate one-half of the fovea, independent of check size.

The apparatus consisted of photographically reduced checkerboard patterns processed into a set of $35-\mathrm{mm}$ slides, in which check size varied from 4 to 108 min of visual angle on a side. The checkerboard slides were presented in a different random order for each of the five replications for each observer. These slides were inserted into a Maxwellian view optical system utilizing tungsten filament bulbs (Sylvania No. $1133,6 \mathrm{~V}, 3.5 \mathrm{~A}$ ), and subtended a circular field size of $7 \mathrm{deg}$. Light was gated temporally by means of a rotating sector disk, which produced flashes of $58 \mathrm{msec}$ duration at a rate of $1 \mathrm{~Hz}$. The retinal illumination produced was 23.8 trolands. The focus was rigorously maintained from one slide's insertion into the optical system to the next. A positionable dental impression bite bar maintained the-observer's 

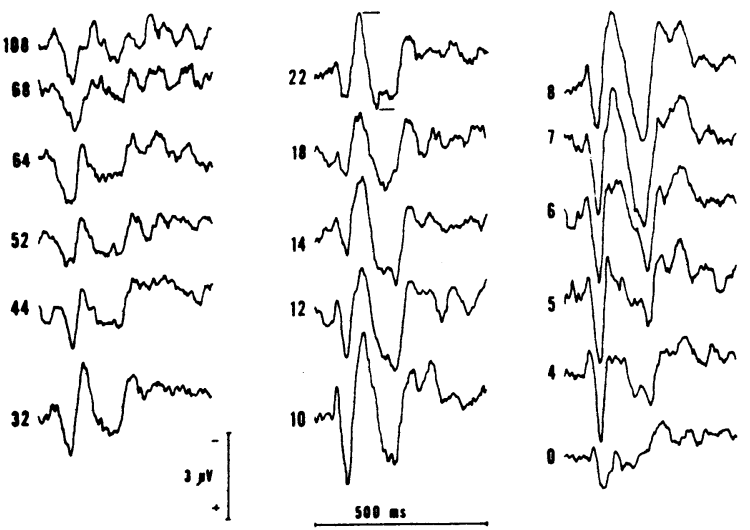

Figure 1. A selected, but typical, sample of VECP waveforms elicited by the set of checkerboard patterns. Amplitude and time calibrations are indicated on the figure. Numbers at the left of each tracing indicate the visual angle in minutes subtended by a side of one check in the pattern. " $O$ " indicates the total absence of a checkerboard pattern. The field intensity was also reduced by $\mathbf{5 0 \%}$ in this condition. Horizontal lines on the uppermost waveform in the center column indicate the feature whose amplitude was measured. Each VECP is the mean of 64 responses.

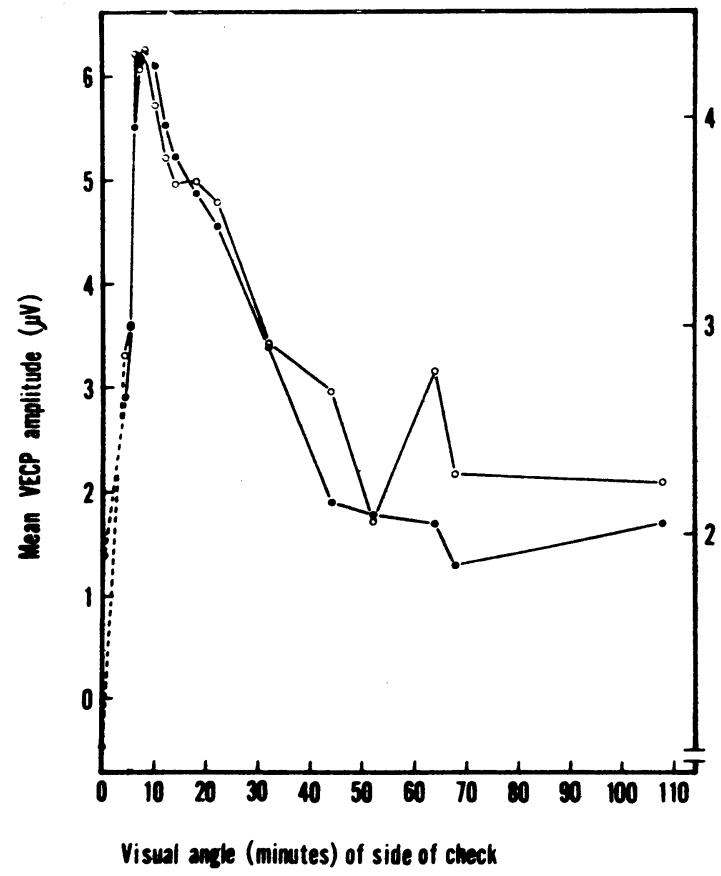

Figure 2. VECP amplitude as a function of checkerboard pattern check size. Amplitudes indicated for $0 \mathrm{~min}$ of visual angle indicate the response to a blank white field, with intensity reduced by $50 \%$. Each point for both observer D.W. (open circles) and J.S. (closed circles) represents the mean of five replications. Note that the ordinates for observer D.W. (on the right of the figure) and for J.S. (on the left of the figure) are scaled differently.

head in proper alignment with the optical system. The patterns were viewed by the right eye, the left eye being covered by an eye-patch. Electrical activity was led off from the scalp by means of a Grass EEG electrode mounted in a holder of my design, positioned in the midline approximately $1 \mathrm{~cm}$ above the inion, and referenced to the right earlobe. The left earlobe was grounded. Scalp potentials were amplified by Tektronix Type 122 low-level preamplifiers, and were summed by a Fabritek Model 1062 instrument computer. The number of responses summed was 64 . The computer was triggered by means of a photodiode circuit which was actuated by a small amount of light deflected from the stimulus beam. Summed potentials were read out by an X-Y plotter.

Figure 1 shows a selected set of VECP waveforms evoked by checkerboard patterns of various sized checks. The feature of the waveform whose amplitude was measured is indicated in the figure, and is seen to be a late negative-positive component, having an implicit time of approximately $140-250 \mathrm{msec}$ following stimulus onset.

Figure 2 shows results for each of the two observers. As check size is decreased (from right to left on the graph), the VECP amplitude rises with generally positive acceleration until a rather sharp peak is reached at a check size of $8 \mathrm{~min}$ for both observers. With still smaller checks, the VECP amplitude drops precipitously. The means and standard errors for each observer can be seen in Table 1 .

These results confirm, in the main, the experiments of Rietveld et al. (1967) and others, who have shown that the VECP amplitude passes through a maximum in response to a series of check sizes. The appearance of the peak at $8 \mathrm{~min}$ is at a smaller check size than that seen in most of the reported experiments, but the exact location of the peak is affected by variables such as sharpness of focus of the checkerboard pattern, for

Table 1

\begin{tabular}{|c|c|c|c|c|}
\hline \multirow{2}{*}{$\begin{array}{l}\text { Check } \\
\text { Size* }\end{array}$} & \multicolumn{2}{|c|}{$\begin{array}{l}\text { Mean Peak-to-Trough } \\
\text { Amplitude }(\mu \mathrm{V})\end{array}$} & \multicolumn{2}{|c|}{ Standard Error } \\
\hline & J.S. & D.W. & J.S. & D.W. \\
\hline $0 \dagger$ & 1.41 & 0.97 & 0.16 & 0.10 \\
\hline 4 & 2.92 & 2.86 & 0.20 & 0.23 \\
\hline 5 & 3.62 & 3.00 & 0.44 & 0.51 \\
\hline 6 & 5.52 & 4.32 & 0.33 & 0.35 \\
\hline 7 & 6.21 & 4.23 & 0.47 & 0.36 \\
\hline 8 & 6.23 & 4.33 & 0.43 & 0.05 \\
\hline 10 & 6.10 & 4.07 & 0.38 & 0.29 \\
\hline 12 & 5.55 & 3.81 & 0.27 & 0.18 \\
\hline 14 & 5.23 & 3.68 & 0.47 & 0.24 \\
\hline 18 & 4.88 & 3.70 & 0.80 & 0.33 \\
\hline 22 & 4.56 & 3.59 & 0.37 & 0.28 \\
\hline 32 & 3.38 & 2.92 & 0.35 & 0.13 \\
\hline 44 & 1.90 & 2.68 & 0.30 & 0.19 \\
\hline 52 & 1.78 & 2.06 & 0.22 & 0.25 \\
\hline 64 & 1.71 & 2.78 & 0.21 & 0.27 \\
\hline 68 & 1.29 & 2.29 & 0.24 & 0.14 \\
\hline 108 & 1.70 & 2.25 & 0.35 & 0.21 \\
\hline
\end{tabular}

*Minutes visual angle
†Blank field 
example, which would be expected to vary from one laboratory to another. It could also be the case that eye movements during an averaging run, which would be likely to occur in the absence of fixation instructions, would serve to blur the image in the case of those flashes which occur during an eye movement.

The close agreement of these data with those of Rietveld et al. (1967) indicates that virtually the same shaped function is obtained when rigorous control of fixation is maintained. However, it is impossible to assess the possible effects of eye movements, or of location of fixation point with respect to the pattern, upon the optimal check size for eliciting the VECP when these experimental details are not reported.

\section{REFERENCES}

CiganeK, L. Binocular addition of the visual response evoked by dichoptic patterned stimuli. Vision Research, 1971, 11, 1289-1297.
HARTER, M. R. Visually evoked cortical responses to the onand off-set of patterned light in humans. Vision Research, 1971, 11, 685-695.

HARTER, M. R., \& White, C. T. Effects of contour sharpness and check-size on visually evoked cortical potentials. Vision Research, 1968, 8, 701-712.

Harter, M. R., \& White, C. T. Evoked cortical responses to checkerboard patterns: Effect of check-size as a function of visual acuity. Electroencephalography and Clinical Neurophysiology, 1970, 28, 48-54.

Rietveld, W. J., Tordoir, W. E. M., Hagenouw, J. R. B., LubBers, J. A., \& Spoor, A. C. Visual evoked responses to blank and to checkerboard patterned flashes. Acta Physiologica and Pharmacologica, Neerl., 1967, 14, 259-285.

Spenlmann, R. The averaged electrical responses to diffuse and to patterned light in the human. Electroencephalography and Clinical Neurophysiology, 1965, 19, 560-569.

(Received for publication June 16, 1975.) 